protein expression, or through association with the rs2294008 $(\mathrm{C}>\mathrm{T})$ polymorphism in the PSCA gene. From in vitro data, this polymorphism appears to be functional, and the risk allele $(T)$ has been shown to be associated with gastric cancer risk in Asians and white individuals. Our study aimed to test for associations between the rs2294008 polymorphism, or PSCA protein expression, and risk of adenomatous polyps and colorectal cancer.

Methods Between 2008 and 2010, we recruited individuals who had tested positively for faecal occult blood, and had undergone colonoscopic screening. Genomic DNA samples were available from 388 subjects with histologically-proven colorectal neoplasia and 493 subjects with no evidence of neoplasia. Genotyping for the rs2294008 polymorphism was performed using a pre-designed TaqMan ${ }^{\circledR}$ assay and the ABI $7900 \mathrm{HT}$ Fast Sequence Detection System. Immunohistochemical (IHC) staining for the PSCA protein was performed using normal and neoplastic tissues covering all stages of the adenoma-carcinoma sequence. The tissue set included adenomatous polyps displaying low-grade $(n=10)$, and high-grade $(n=10)$ epithelial dysplasia, and adenomatous polyps harbouring invasive carcinoma $(n=10)$. Normal adjacent mucosa was assessed in the polyp sections in addition to separate normal mucosal sections $(n=4)$. Positive staining of colonic crypt neuroendocrine cells served as an internal positive control.

Results There was no association between the rs2294008 SNP and risk of colorectal neoplasia in either dominant (OR 0.97; 95\% CI 0.73 to 1.28 ) or recessive (OR 0.88 ; $95 \%$ CI 0.61 to 1.27 ) genotype models. IHC analysis of colonic tissue samples indicated no alteration in the topographic distribution or intensity of PSCA staining between normal mucosa, adenomatous mucosa with low or high grade epithelial dysplasia, and invasive carcinoma.

Conclusion Our results suggest that PSCA does not play an important role in the initiation or progression of colorectal carcinogenesis. Given that PSCA has been implicated in a variety of other solid tumours, continued efforts should be made to elucidate the normal and pathological cellular functions of PSCA.

Competing interests None declared.

\section{PWE-076 CONTINUED BIENNIAL SCREENING OF FAECAL OCCULT BLOOD TEST (FOBT) POSITIVE AND SCREENING COLONOSCOPY NEGATIVE COHORT IN ENGLISH BOWEL CANCER SCREENING PROGRAMME-IS IT NECESSARY?}

doi:10.1136/gutjnl-2012-302514d.76

${ }^{1} \mathrm{D}$ Majumdar, * $2 \mathrm{~J}$ Patnick, ${ }^{2} \mathrm{C}$ Nickerson, ${ }^{1} \mathrm{M} \mathrm{D}$ Rutter. ${ }^{1}$ Department of Gastroenterology, University Hospital of North Tees, Stockton on Tees, UK; ${ }^{2}$ NHS Cancer Screening Programmes, NHS Cancer Screening Programmes, Sheffield, UK

Introduction The English NHS Bowel Cancer Screening Programme (BCSP) uses guaiac based faecal occult blood test (FOBT) as the screening tool, with people with a positive result undergoing colonoscopy. Subjects with adenomas who are in the low risk category and those with no adenomas at colonoscopy are invited to participate in the next gFOBT round in 2 years. This study evaluates the PPV of a second FOBT diagnostic colonoscopy following a second FOBT positive result.

Methods Data on each patient entering the NHS BCSP programme is prospectively recorded on the national BCSP database. The database was interrogated to identify subjects who had had a second FOBT positive diagnostic colonoscopy in BCSP 2 years after their first screening colonoscopy. The diagnostic colonoscopy PPV of this second FOB positive procedure was compared with the published $\mathrm{PPV}$ of first $\mathrm{FOB}$ positive diagnostic colonoscopies. ${ }^{1}$

Results The database was interrogated in April 2011. A total of 772 subjects were identified. The positive predictive value (PPV) for all colorectal neoplasia was $25.7 \%(n=199)$ and $0.9 \%(n=7)$ for color- ectal cancer (CRC). 41.5\% had a normal colonoscopy and 32.8\% had non-neoplastic pathology. This compares with a PPV for CRC at the first $\mathrm{FOB}$ positive diagnostic colonoscopy of $10.1 \%$ and for all neoplasia of $53 \% .^{2}$ Findings are summarised in the Abstract PWE076 table 1 below. Out of the seven cancers three were Dukes' C, 2 Dukes' B and 2 Dukes' A stage. The sizes of the cancers ranged from $20 \mathrm{~mm}$ to $60 \mathrm{~mm}$. Three were located in the rectum, three at the recto-sigmoid junction and one in the caecum.

Abstract PWE-076 Table 1 Outcome of 1st and 2nd FOBT positive colonoscopies

\begin{tabular}{|c|c|c|c|c|c|}
\hline Screening cycle & $\begin{array}{l}\text { Total } \\
\text { number }\end{array}$ & Cancer & High risk & $\begin{array}{l}\text { Intermediate } \\
\text { risk }\end{array}$ & Low risk \\
\hline $\begin{array}{l}\text { First FOB } \\
\text { colonoscopy }\end{array}$ & 17518 & $\begin{array}{l}1772 \\
(10.1 \%)\end{array}$ & $\begin{array}{l}1721 \\
(9.8 \%)\end{array}$ & $\begin{array}{l}3050 \\
(17.4 \%)\end{array}$ & $\begin{array}{l}2743 \\
(15.7 \%)\end{array}$ \\
\hline $\begin{array}{l}\text { Second FOB } \\
\text { colonoscopy }\end{array}$ & 772 & $7(0.9 \%)$ & $7(0.9 \%)$ & 41 (5.31\%) & $144(18.7 \%)$ \\
\hline $\begin{array}{l}\mathrm{p} \text { Value (Fisher's } \\
\text { exact, } 2 \text { tailed) }\end{array}$ & & $<0.0001$ & $<0.0001$ & $<0.0001$ & 0.51 \\
\hline
\end{tabular}

Conclusion There is significant reduction of CRC and adenoma in the population undergoing a second FOBT positive colonoscopy compared to the first one ( $0.9 \%$ vs $10.1 \%$, p value $<0.0001$ for CRC). Though the numbers are small, in the cohort where cancer is detected, presence of locally advanced cancer raises the question of missed lesion during the colonoscopy after first positive FOBT and therefore the current practise of biennial FOBT screening for this group is justified.

Competing interests None declared.

\section{REFERENCE}

1. Logan R, Patnick J, Nickerson C, et al. Outcome of the Bowel Cancer Screening Programe in England after the first 1 million tests. Gut. Published Online First: 7 December 2011. doi:10.1136/gutjnl-2011-300843

\section{PWE-077 PILOT OF FLEXIBLE SIGMOIDOSCOPY SCREENING TO PREVENT COLORECTAL CANCER}

doi:10.1136/gutjnl-2012-302514d.77

${ }^{1}$ E Sofianopoulou, ${ }^{* 1} \mathrm{G}$ Rubin, ${ }^{2} \mathrm{M}$ Ritchie, ${ }^{3} \mathrm{~J}$ Patnick, ${ }^{1,2,4} \mathrm{C}$ Rees. ${ }^{1}$ School of Medicine and Health, Durham University, Stockton-On-Tees, UK; ${ }^{2}$ South of Tyne Bowel Cancer Screening Centre, District Hospital, South Tyneside, UK; ${ }^{3}$ NHS Cancer Screening Programmes, Sheffield, UK; ${ }^{4}$ Northern Region Endoscopy Group, UK

Introduction A screening programme in England to prevent colorectal cancer using flexible sigmoidoscopy (FSIG) was announced in late 2010, following the results of a major UK study showing that a one-off FSIG offered to people aged 55-64 years significantly reduced colorectal cancer incidence and mortality. Three "pathfinder" sites, in Derby, South of Tyne and Tees, were selected to assess the practicalities of invitation and FSIG screening. We report the findings of our evaluation of this pathfinder phase.

Methods Patients aged 55 yrs and registered with one of 31 selected practices in three pathfinder areas received postal invitations to participate. The South of Tyne and Derby sites employed similar, interactive model of screening invitation involving telephone preassessment by specialist screening practitioners, while Tees used a simple invitation. We used routinely collected data to assess screening uptake, process and outcomes. A self-completion patient satisfaction questionnaire was sent 1-month after attendance to all participants. A postal questionnaire was sent to the 31 participating GP practices that had been selected to participate. Screening took place for a 3-month period in early 2011. 
Results In total 4022 people aged 55 years were invited and 1129 $(28 \%)$ screened over the 3-month period. Screening uptake differed by method of invitation, with a simple approach being significantly more successful than one that was more interactive (32\% vs $27 \%$, $\mathrm{p}=0.0015)$. Uptake also decreased significantly with increasing deprivation. Adenomas were found in 111 (9.8\%) of those screened. Cancer was found in two subjects. Over $95 \%$ of participants rated the procedure as "very" or "fairly" acceptable. Over $90 \%$ of respondents said they would participate in cancer screening in the future and a similar proportion would recommend doing so to others. 11 out of 31 practices offered an estimate of the time spent on activities relating to this pathfinder for FSIG screening, most giving a figure of $<1 \%$.

Conclusion In this pathfinder study, a simple invitation to FSIG achieved a greater uptake rate than a more complex, interactive method of invitation. Deprivation was associated with lower screening uptake. Further pilots of the flexible sigmoidoscopy screening programme should focus on strategies to increase response rate and to improve technical aspects of the procedure. Further evaluation should be incorporated into that development.

Competing interests None declared.

\section{PWE-078 INCIDENCE AND MANAGEMENT OF MALIGNANT POLYPS IN THE NORTH ESSEX BOWEL CANCER SCREENING PROGRAMME}

doi:10.1136/gutjnl-2012-302514d.78

E Paulon,* D O'Riordan, A Shenoy. Department of Gastroenterology, Colchester General Hospital, Colchester, UK

Introduction The outcome of colonic cancer in the UK population has sensibly improved since the introduction of the Bowel Cancer Screening Programme (BCSP) in 2006. This is mainly due to early detection of the cancer in its early stages of which the malignant polyp (MP) is often a manifestation. In this study we reviewed the detection rates and management of malignant polyps and their outcomes in the population of north and mid Essex and compared it to national and international experience.

Methods 33 patients with malignant polyps were identified between the start of the North Essex screening programme (February 2009) and December 2010. Data were obtained using patient notes, endoscopy, radiology and histopathology reports.31 patients were included in the study because data of two patients were not available.

Results Of the 31 polyps, the vast majority (94\%) was located in the left colon. $19(61 \%)$ were peduncolated. Polyps average size was $1.5 \mathrm{~cm}, 8(26 \%)$ measured $>2 \mathrm{~cm}$ and only $2(6 \%)$ measured $<1 \mathrm{~cm}$. Haggitt and Kikuchi classifications were used to assess the depth of invasion. Patients were divided into low risk (LR) and high risk (HR) groups according to histologic criteria (depth of invasion, lymphovascular invasion, grade and excision margins $>2 \mathrm{~mm}$ ). 16 (52\%) fell into the LR group, 15 (48\%) were considered at high risk. 29 (94\%) patients had staging CT, with an average waiting time of 3.5 weeks for patients at HR (range 2-13 weeks) and 6 weeks for patients at LR (range 2-24 weeks). Five patients were surgically treated within 10 weeks (4 HR, $1 \mathrm{LR}$ ). The remaining patients were followed-up with a repeat endoscopy, with a mean waiting time of 9 weeks for patients at HR (range $4-15$ weeks) and 24 weeks for patients at LR (range 9-60 weeks). Residual tumour was identified on endoscopic follow-up in two patients at HR. Surgery was finally performed in 15 patients (48.5\%), $13 \mathrm{HR}, 2 \mathrm{LR}$. Lymph nodes (LNs) involvement was present in four patients (30\%). Residual tumour was found in 5 (33\%). Surgical complications occurred in two patients $(13 \%)$ and consisted in one anastomotic leak and one bilateral pulmonary embolism. To date cancer recurrence has been found in $2 \mathrm{HR}$ patients $(6.5 \%)$ who had evidence of LNs involvement (median follow-up of 16 months, range 8-28).

Conclusion CT scans and endoscopic follow-up timing varied greatly between patients, especially for those in the LR group. LNs involvement and recurrence rate $(30 \%)$ was higher than described in London BCSP centres (9.8\%) but still consistent with other published data. ${ }^{1}$ Further research is needed to clarify the need for staging CT and timing of follow-up endoscopy in patients at LR.

Competing interests None declared.

\section{REFERENCE}

1. Hassan C, Zullo A, Winn S, et al. The colorectal malignant polyp: scoping a dilemma Dig Liver Dis 2007;39:92-100.

\section{PWE-079 CHARACTERISATION COLONIC POLYPS USING FICE WITHOUT OPTICAL MAGNIFICATION: A NEW CLASSIFICATION SYSTEM (N.A.C.)}

doi:10.1136/gutjnl-2012-302514d.79

G Longcroft-Wheaton,* P Bhandari. Department of Gastroenterology, Portsmouth Hospitals NHS Trust, Portsmouth, UK

Introduction FICE is a novel method of electronic imaging on fujinon endoscopes which enhances surface and vascular patterns. It can be used to predict histology of colonic polyps. However, there are no validated classification systems for using the technique without magnification. This study aims to validate a novel tool for this purpose.

Methods The optimum FICE setting was determined in a picture study. Digital photographs were taken of polyps with white light and all 10 FICE settings at $1026 \times 770$ resolution. They were reviewed by two endoscopists who scored each image for clarity of the mucosal and vascular patterns on a 3 point scale $(0=$ poor, $3=$ excellent). A second library was examined to determine the criteria associated with hyperplastic polyps, adenomas and cancer. In each case the true histology was known. A prospective study was then performed to test the identified characteristics in vivo. Finally the criteria were used in a prospective study of polyps $<10 \mathrm{~mm}$ in size within a screening population.

\section{Abstract PWE-079 Table 1}

\begin{tabular}{|c|c|c|c|c|c|c|}
\hline & \multicolumn{2}{|c|}{ Hyperplastic } & \multicolumn{2}{|c|}{ Adenomas } & \multicolumn{2}{|l|}{ Cancers } \\
\hline & Study 2 & Study 3 & Study 2 & Study 3 & Study 2 & Study 3 \\
\hline \multicolumn{7}{|l|}{ WLI features (vascularity) } \\
\hline Pale & $83 \%$ & $59 \%$ & $22 \%$ & $17 \%$ & $0 \%$ & $0 \%$ \\
\hline Normal & $0 \%$ & $4 \%$ & $0 \%$ & $13 \%$ & $0 \%$ & $0 \%$ \\
\hline Dark & $17 \%$ & $37 \%$ & $78 \%$ & $70 \%$ & $100 \%$ & $100 \%$ \\
\hline \multicolumn{7}{|l|}{ FICE features (vascularity) } \\
\hline Pale & $80 \%$ & $67 \%$ & $6 \%$ & $17 \%$ & $0 \%$ & $0 \%$ \\
\hline Dark & $20 \%$ & $33 \%$ & $94 \%$ & $83 \%$ & $0 \%$ & $0 \%$ \\
\hline Very dark & $0 \%$ & $0 \%$ & $0 \%$ & $0 \%$ & $100 \%$ & $100 \%$ \\
\hline \multicolumn{7}{|l|}{ FICE features (vascular patterns) } \\
\hline Absent vascular pattern & $50 \%$ & $48 \%$ & $0 \%$ & $13 \%$ & $0 \%$ & $0 \%$ \\
\hline Faint vessels not following crypts & $50 \%$ & $35 \%$ & $0 \%$ & $1 \%$ & $0 \%$ & $0 \%$ \\
\hline Regular pericryptal pattern & $0 \%$ & $17 \%$ & $94 \%$ & $86 \%$ & $0 \%$ & $0 \%$ \\
\hline Dense, irregular pattern & $0 \%$ & $0 \%$ & $0 \%$ & $0 \%$ & $100 \%$ & $100 \%$ \\
\hline \multicolumn{7}{|l|}{ FICE surface pattern } \\
\hline No surface pattern & $63 \%$ & $37 \%$ & $0 \%$ & $0 \%$ & $0 \%$ & $0 \%$ \\
\hline Large, non-compact crypt pattern & $37 \%$ & $39 \%$ & $0 \%$ & $9 \%$ & $0 \%$ & $0 \%$ \\
\hline Small, compact, regular pattern & $0 \%$ & $17 \%$ & $88 \%$ & $88 \%$ & $0 \%$ & $0 \%$ \\
\hline Disorganised, irregular pattern & $0 \%$ & $0 \%$ & $0 \%$ & $0 \%$ & $100 \%$ & $100 \%$ \\
\hline Cannot assess & $0 \%$ & $7 \%$ & $12 \%$ & $3 \%$ & $0 \%$ & $0 \%$ \\
\hline
\end{tabular}

